\title{
Retrograde Suction Decompression for Clip Occlusion of Internal Carotid Artery Communicating Segment Aneurysms
}

\section{Takeuchi, Satoru}

2016-05

Takeuchi , S , Tanikawa , R , Goehre , F , Hernesniemi , J , Tsuboi , T , Noda , K, Miyata , S , Ota , N , Sakakibara , F , Andrade-Barazarte , H \& Kamiyama , H 2016 , ' Retrograde Suction Decompression for Clip Occlusion of Internal Carotid Artery Communicating Segment Aneurysms ' , World Neurosurgery , vol. 89 , pp. 19-25 . https://doi.org/10.1016/j.wneu.2015.12.095

http://hdl.handle.net/10138/224039

https://doi.org/10.1016/j.wneu.2015.12.095

publishedVersion

Downloaded from Helda, University of Helsinki institutional repository.

This is an electronic reprint of the original article.

This reprint may differ from the original in pagination and typographic detail.

Please cite the original version. 


\section{Retrograde Suction Decompression for Clip Occlusion of Internal Carotid Artery Communicating Segment Aneurysms}

\section{Satoru Takeuchi ${ }^{1,2}$, Rokuya Tanikawa ${ }^{2}$, Felix Goehre ${ }^{3}$, Juha Hernesniemi ${ }^{3}$, Toshiyuki Tsuboi ${ }^{2}$, Kosumo Noda $^{2}$ Shiro Miyata ${ }^{2}$, Nakao Ota $^{2}$, Fumihiro Sakakibara ${ }^{2}$, Hugo Andrade-Barazarte ${ }^{3}$, Hiroyasu Kamiyama ${ }^{2}$}

BACKGROUND: Retrograde suction decompression (RSD) can achieve proximal parent vessel control, improve aneurysm neck visualization, and allow parent vessel reconstruction for direct clipping of internal carotid artery (ICA) aneurysms. The aim of the present study was to describe the technique and surgical results of RSD for direct clipping of ICA communicating segment (C1) aneurysms.

METHODS: The clinical data and treatment summaries of 20 patients who underwent RSD-assisted clipping of ICA C1 aneurysms were retrospectively reviewed. Pre- and postoperative three- or four-dimensional computed tomography angiograms, postoperative magnetic resonance images, surgical notes, operative complications, and outcomes were assessed.

RESULTS: All patients except one harbored unruptured C1 aneurysms. Extracranial-intracranial graft bypass using the radial artery was performed in five patients. Fifteen patients required temporary clipping of the posterior communicating artery for further reduction of blood back-flow into the aneurysm. All aneurysms were successfully clipped and postoperative three- or four-dimensional computed tomography angiography revealed no major branch occlusion or residual aneurysm. At the 6-month follow-up examination, 19 patients had a good outcome and 1 patient had poor outcome associated with anterior choroidal artery ischemia. No death had occurred at 6-month follow-up examination.
CONCLUSIONS: The RSD technique is a useful procedure to achieve proximal vascular control, to soften and shrinkage the aneurysm sac, and to provide a wide and clean operative field allowing safe clip placement. The RSD technique requires special attention to the relationship between the perforators and the aneurysm, and close cooperation between the surgeon and the assistant.

\section{INTRODUCTION}

A neurysms arising from the internal carotid artery (ICA) are common and usually located at the communicating segment of the ICA (CI). ${ }^{\mathrm{I}, 2}$ These CI aneurysms frequently involve the origin of the posterior communicating artery (PcomA) and anterior choroidal artery (AchA). In addition, some CI aneurysms tend to adhere to the surrounding structures such as the AchA, anterior thalamoperforating artery (ATPA), oculomotor nerve, and the dura of the skull base. Proximal temporary clipping is often used to reduce the transmural pressure into the parent vessel and to soften the aneurysm sac. In some circumstances, the aneurysm remains tense after proximal and distal temporary clipping of the ICA, leading to difficulties for permanent clip placement, such as clip slipping or occlusion of the parent artery and perforators.

The suction decompression technique using direct aneurysmal puncture into giant aneurysms was introduced in $198 \mathrm{r} .^{3}$ Batjer

mRS: Modified Rankin Scale

PcomA: Posterior communicating artery

RA: Radial artery

RSD: Retrograde suction decompression

STA: Superficial temporary artery

From the ${ }^{\mathbf{1}}$ Department of Neurosurgery, National Defense Medical College, Tokorozawa Saitama, Japan; ${ }^{2}$ Department of Neurosurgery, Teishinkai Hospital, Sapporo, Hokkaido, Japan; and ${ }^{\mathbf{3}}$ Department of Neurosurgery, Helsinki University Hospital, Helsinki, Finland

To whom correspondence should be addressed: Satoru Takeuchi, M.D.

[E-mail: s.takeuchi@room.ocn.ne.jp]

Citation: World Neurosurg. (2016) 89:19-25.

http://dx.doi.org/10.1016/j.wneu.2015.12.095

Journal homepage: www.WORLDNEUROSURGERY.org

Available online: www.sciencedirect.com

1878-8750/\$ - see front matter (c) 2016 Elsevier Inc. All rights reserved. 
and Samson ${ }^{4}$ in 1990 and Tamaki et al. ${ }^{5}$ in I99I described the surgical retrograde suction decompression (RSD) technique as an alternative to achieve proximal vascular control of the parent vessel, to improve the visualization of the aneurysm neck, and to reconstruct the parent vessel without occluding important perforators. ${ }^{\mathrm{I} 4^{-12}}$ Subsequently, the adoption of less invasive procedures has led to the use of the endovascular suction decompression technique as an option to surgical exposure; however, it carries a higher risk of ICA dissection. ${ }^{1,6-9,12}$ The surgical RSD technique has been applied mainly for large or giant aneurysms located in the ophthalmic $\left(\mathrm{C}_{2}\right)$ /clinoid $\left(\mathrm{C}_{3}\right)$ segment of the ICA, and less often for Ci aneurysms. ${ }^{13}$ Previous case series of RSD-assisted clipping focused mainly on $\mathrm{C}_{2} / \mathrm{C}_{3}$ aneurysms or failed to show the results of RSD-assisted clipping for CI aneurysms. ${ }^{6,7}$

The present study analyzed 20 consecutive patients with $\mathrm{CI}$ aneurysms treated through direct microsurgical clipping assisted by RSD to provide proximal control of the parent vessel to evaluate the surgical technique and feasibility of RSD for direct clipping of CI aneurysms.

\section{METHODS}

\section{Study Cohort}

This study retrospectively analyzed 338 patients with ICA aneurysms treated at our institution between April 2012 and March 2015. This study excluded the 157 patients with $\mathrm{C}_{2} / 3$ aneurysms, 2I patients with $\mathrm{C}_{4}$ aneurysms, and 140 patients who underwent only clipping, clipping with bypass, or trapping with bypass without RSD for CI aneurysms. Finally, this study included only 20 patients who underwent RSD-assisted direct microsurgical clipping of $\mathrm{CI}$ aneurysms. The study protocol was approved by the institutional ethics committee.

\section{Clinical and Radiological Data}

The clinical charts and treatment summaries of the 20 patients were analyzed retrospectively to identify associated comorbidities and preoperative clinical status. Each patient underwent preoperative three- and four-dimensional computed tomography (3D-CT and ${ }_{4} \mathrm{D}-\mathrm{CT}$ ) angiography to assess parent vessel characteristics (e.g., presence of calcifications, diameter), aneurysm characteristics (e.g., shape, wall features, neck diameter, size), relationships between the aneurysm and vessel branches, and bony structures to determine the best surgical strategy. $4 \mathrm{D}$-CT angiography was performed if $3 \mathrm{D}$-CT angiography failed to demonstrate the AchA or ATPA (Figure 1). We do not routinely perform preoperative digital subtraction angiography for intracranial aneurysms at our institution.

\section{Outcome}

All patients underwent direct surgical treatment of the CI aneurysm. Clinical outcome according to the modified Rankin Scale (mRS) was assessed by a neurosurgeon immediately after surgery and at 6 months follow-up. Outcome was classified according to the mRS score as good $(0-2)$ or poor $(3-6)$. $3 \mathrm{D}$ - or $4 \mathrm{D}-\mathrm{CT}$ angiography and magnetic resonance imaging were performed after the surgical procedure to evaluate acute complications, patency of the parent vessel, and aneurysm occlusion.

\section{Surgical Strategy}

The indications to perform surgical RSD were based on adhesion of the aneurysm dome or neck, or both, to the PcomA, ATPA, and AchA in preoperative studies, regardless of the size of the aneurysm. The first intention to treat $\mathrm{C}_{\mathrm{I}}$ aneurysms was direct clipping if possible. However, CI aneurysms with complex anatomy (perforators arising from the aneurysm sac, presence of calcifications) and large/giant size were considered unclippable and were treated by trapping and bypass. The bypass indications were based on the complexity of the aneurysm (size, projection, and relationship between the aneurysm and vessel branches).

The patients were divided into two groups according to the surgical strategy. Group I included patients with aneurysm clipping requiring short temporary parent vessel occlusion time $(<$ IO min) and who underwent only RSD-assisted clipping. Group 2 included patients with aneurysm clipping requiring long temporary parent vessel occlusion time ( $\geq$ IO $\mathrm{min}$ ) and who underwent RSD-assisted clipping and superficial temporary artery (STA)middle cerebral artery (MCA) bypass, external carotid artery (ECA)-radial artery (RA)-M2 bypass, or both.

\section{Surgical Technique: RSD-Assisted Clipping and Bypass}

Figure 2 shows a schematic drawing of the RSD technique. The patient was positioned supine with the head rotated 45 degrees to the opposite side. Intraoperative transcranial motor-evoked potentials and somatosensory-evoked potentials were monitored. The first step corresponded to neck dissection under the operating microscope, followed by identification and exposure of the common carotid artery (CCA), ICA, ECA, and superior thyroid artery. Next, multiple vessel loops were placed around the CCA, ICA, and ECA. If necessary, STA-MCA bypass and/or ECA-RA-M2 bypass was performed before approaching the aneurysm. ${ }^{13}$

All patients underwent an anterior temporal approach as described previously. ${ }^{\text {I4, I5 }}$ After preliminary exposure of the aneurysm, the RSD technique was initiated. In Group I (patients without ECA-RA-M2 bypass), the superior thyroid artery was incised, and a polyvinyl chloride catheter (Atom indwelling feeding tube for infants, $5 \mathrm{Fr}$; Atom Medical, Tokyo, Japan) was introduced into the ICA through the superior thyroid artery (Figure 3A). ${ }^{5}$ In Group 2 (patients with ECA-RA-M2 bypass), the ICA was punctured with a I8-gauge indwelling needle (Figure 3B). After these preparations, temporary clamps and clips were placed on the CCA and the ECA, followed by clamps on the intracranial ICA ( $\mathrm{CI}_{\mathrm{I}}$ segment) distal to the aneurysm neck (Figure 4A, B), or on the $A I$ and Mr. In patients with a large PcomA, a temporary clip was applied to reduce blood back-flow into the aneurysm (Figure 4C). Subsequently, blood was gently aspirated through a tube or needle introduced into the cervical ICA, resulting in aneurysm collapse and enabling the surgeon to complete aneurysm dissection and clipping with preservation of the PcomA, ATPA, and AchA (Figure 4D-F). The occlusion time was limited to ro minutes per attempt in patients without bypass to prevent cerebral ischemia; therefore, RSD was staged in some cases. After aneurysm clipping, the temporary clamps and clips were removed. Finally, micro-Doppler ultrasonography and indocyanine green videoangiography were used to confirm successful clipping and patency of the parent vessel. 

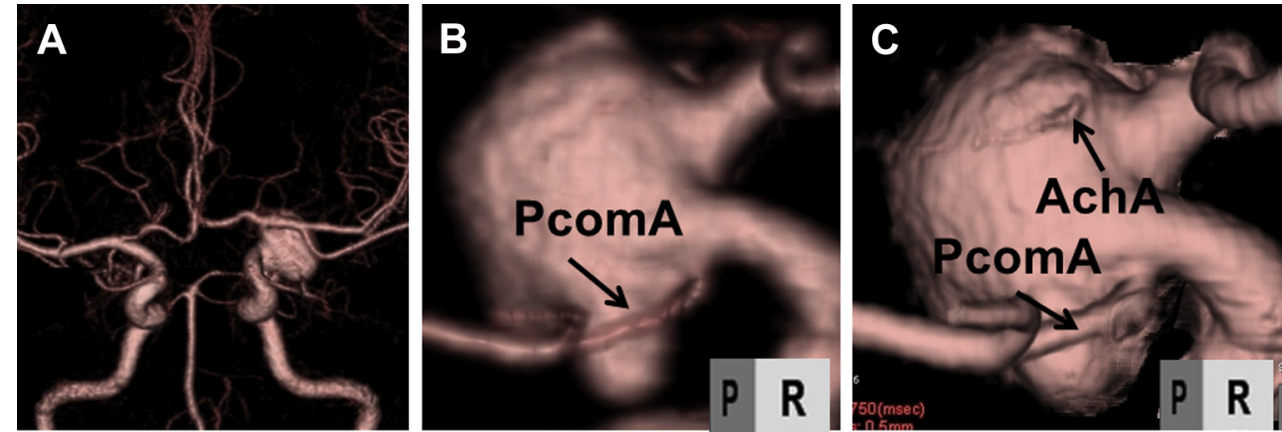

Figure 1. Representative preoperative computed tomography angiograms (Case 8). Three-dimensional computed tomography angiograms from the $(\mathbf{A})$ anteroposterior view and (B) posterolateral view show a large left $\mathrm{C} 1$ aneurysm $(18 \mathrm{~mm}$ in diameter). Note that the anterior choroidal artery (AchA) is not visible. (C) Four-dimensional computed tomography angiogram (posterolateral view) revealing that the AchA originates from the aneurysm dome. PcomA, posterior communicating artery.

\section{RESULTS}

\section{Preoperative Clinical Data and Aneurysm Characteristics}

Nineteen patients had unruptured aneurysms, and I patient had a ruptured aneurysm presenting with Hunt and Hess grade 2. Fifteen of the unruptured aneurysms were incidental findings, whereas 2 manifested as preoperative visual deficits, 2 as chronic headaches, and I with vertigo. All aneurysms were saccular without side predominance (Io right versus Io left). The aneurysm size was medium (7-I4 $\mathrm{mm}$ ) for II aneurysms, large (I4-2I mm) for 7 aneurysms, and giant $(>2 \mathrm{I} \mathrm{mm})$ for 2 aneurysms. Table 1 summarizes the clinical data and aneurysm characteristics.

\section{Surgical Treatment and RSD}

Group I included I6 patients treated with RSD and assisted clipping, and Group 2 included 4 patients requiring additional ECARA-M2 bypass. Extradural anterior clinoidectomy was performed

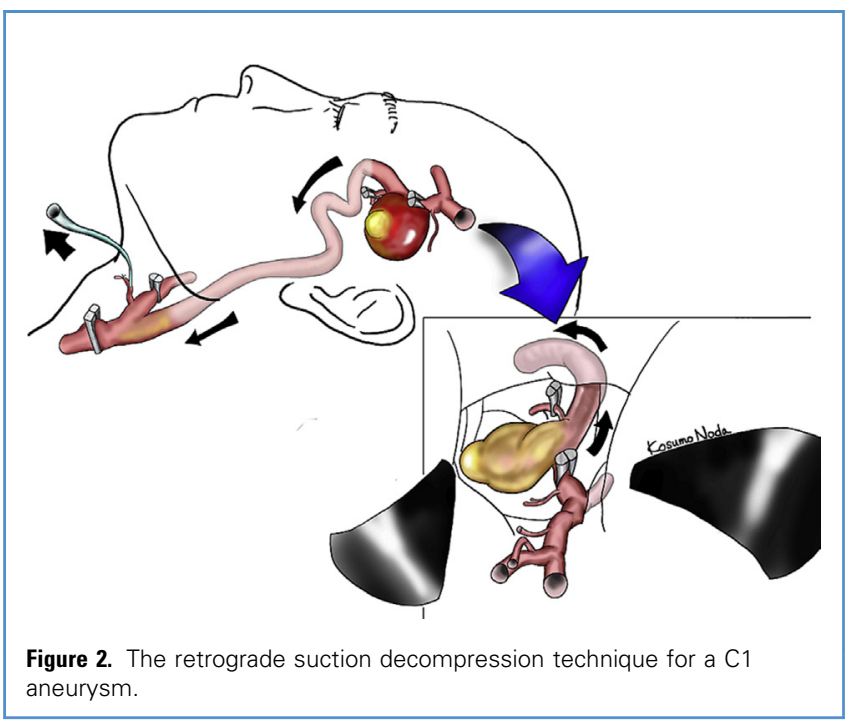

in 6 patients because of the large size of the aneurysm. Distal temporary clipping was performed at the $\mathrm{CI}$ and PcomA in $\mathrm{I3}$ patients, only $\mathrm{CI}_{\mathrm{I}}$ in 3 patients, $\mathrm{AI}, \mathrm{Mr}$, and PcomA in 2 patients, and $\mathrm{AI}$ and $\mathrm{Mr}$ in 2 patients. The total occlusion time ranged from 283 to II26 seconds (mean, 595.3 seconds).

\section{Clinical and Radiological Outcomes}

All aneurysms were successfully treated, and postoperative images demonstrated complete occlusion of the aneurysm and patency of the parent vessel. Postoperative radiologic ischemic events compromising the ATPA or AchA territories appeared in 7 patients (Figure 5). Of these patients, 3 had transient neurologic deficits that had completely resolved by the last follow-up examination, and 2 patients (patients I and I3) had permanent neurologic deficits characterized by hemiparesis, memory disturbances, and mild aphasia. Nineteen patients had good outcome, and I patient had poor outcome associated with AchA ischemia (patient 13) with no surgical mortality at the 6-month follow-up examination.

\section{DISCUSSION}

\section{Advantages of RSD Technique for C1 Aneurysms}

In our series, surgical RSD-assisted clipping allowed for successful surgical management of $\mathrm{CI}_{\mathrm{I}}$ aneurysms, as previously reported. ${ }^{6,7}$ Treatment of Ci aneurysms can be hindered by large aneurysm size, arachnoid adhesions of the aneurysm dome or neck to the surrounding neurovascular structures (PcomA, ATPA, AchA, the oculomotor nerve, and the dura of skull base), or the presence of calcifications on the proximal ICA. The RSD technique provides proximal vascular control of the ICA, and allows softening and shrinking of the aneurysm sac by continuous suction, thus improving the surgical view and visualization of the aneurysm neck and its surrounding structures, and allowing the neurosurgeon to place the permanent clips safely and correctly. ${ }^{6}$ Previously, the surgical RSD technique has been applied mainly for large or giant aneurysms. However, treatment of medium-sized aneurysms that are attached to the PcomA, ATPA, or AchA may benefit from this technique to achieve direct clipping and final occlusion 


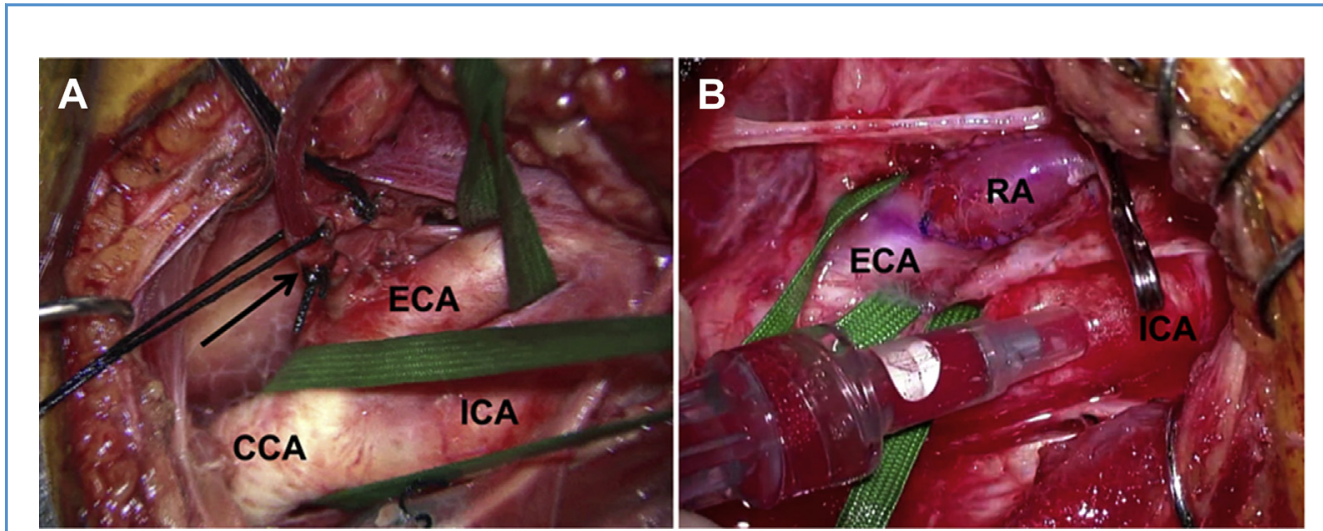

Figure 3. Representative intraoperative photographs (cervical procedure). (A) Case without external carotid artery (ECA)-radial artery (RA)-M2 bypass. The superior thyroid artery (arrow) is incised, and a polyvinyl chloride catheter is introduced into the internal carotid artery (ICA) through the superior thyroid artery. (B) Case with ECA-RA-M2 bypass. The ICA is punctured with an 18-gauge indwelling needle after the bypass procedure. CCA, common carotid artery.

of the aneurysm. Our present study demonstrates that surgical dissection of the neck carries a relatively low risk of permanent morbidity.

\section{Surgical Nuances}

The treatment indications for incidental medium-sized $\mathrm{CI}_{\mathrm{I}}$ aneurysms are based on the risk factors, such as smoking, female sex,

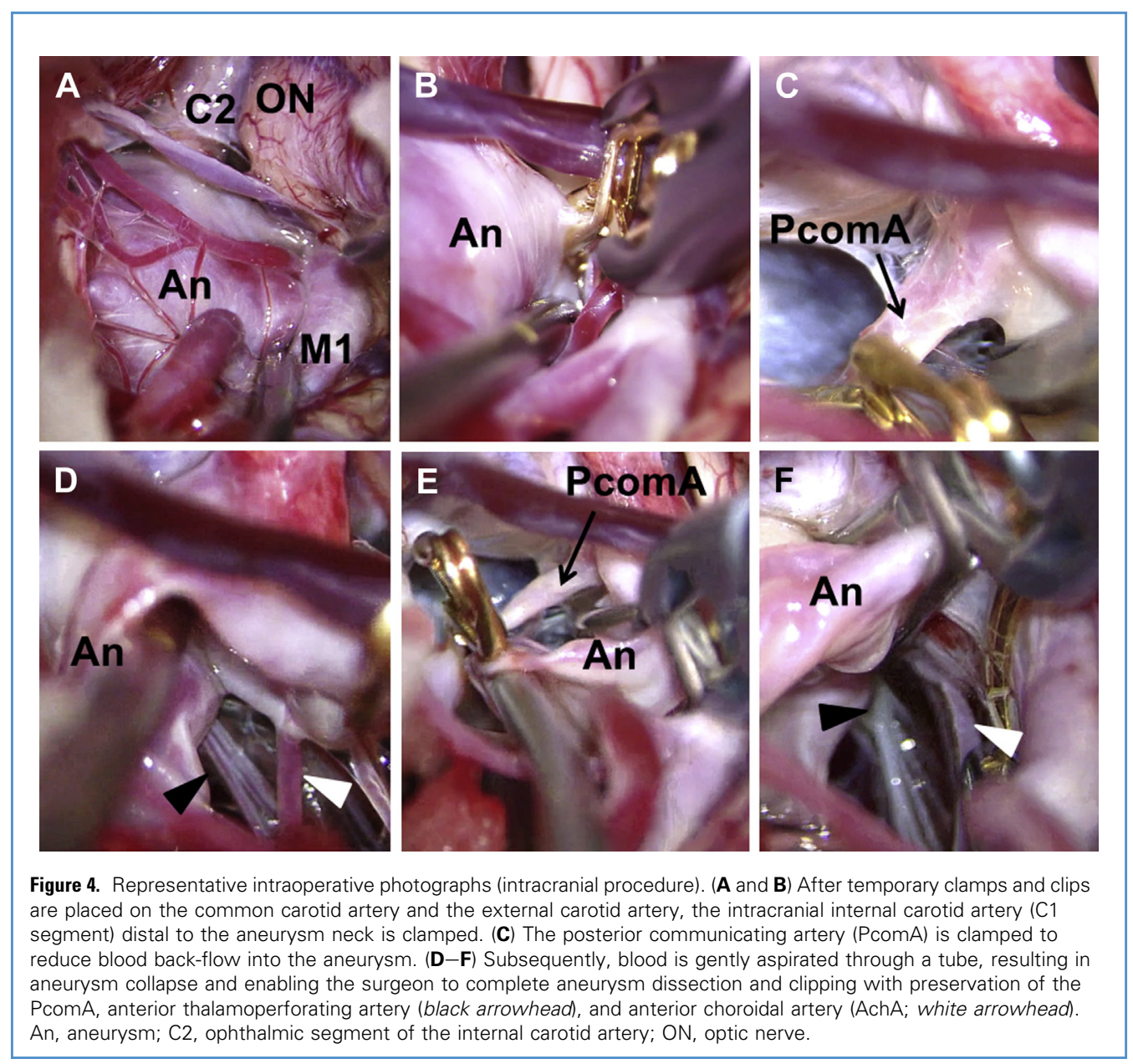


Table 1. Summary of Clinical Data

\begin{tabular}{|c|c|c|c|c|c|c|c|c|c|c|c|c|c|}
\hline $\begin{array}{l}\text { Case } \\
\text { No. }\end{array}$ & $\begin{array}{c}\text { Age } \\
\text { (years) }\end{array}$ & Sex & $\begin{array}{l}\text { Unruptured or } \\
\text { Ruptured }\end{array}$ & Side & $\begin{array}{l}\text { Size } \\
(\mathrm{mm})\end{array}$ & $\begin{array}{l}\text { Additional } \\
\text { Procedure }\end{array}$ & $\begin{array}{l}\text { Fetal } \\
\text { PcomA } \\
\text { Type }\end{array}$ & $\begin{array}{l}\text { Site of Catheter } \\
\text { Insertion }\end{array}$ & $\begin{array}{l}\text { Site of Distal } \\
\text { Temporary } \\
\text { Clip }\end{array}$ & $\begin{array}{l}\text { Total Occlusion } \\
\text { Time for Neck } \\
\text { Clippings (seconds) }\end{array}$ & Complication & $\begin{array}{c}\text { Postoperative } \\
\text { MRI } \\
\text { Findings }\end{array}$ & $\mathrm{mRS}$ \\
\hline 1 & 70 & Female & Unruptured & Left & 20.0 & $\begin{array}{l}\text { Anterior clinoidectomy, } \\
\text { STA-MCA bypass, } \\
\text { STA-P2 bypass, } \\
\text { ECA-RA-M2 bypass }\end{array}$ & Yes & Cervical ICA & C1, PcomA & 684 & Mild aphasia, paresis, ONP & ATPA infarction & 2 \\
\hline 2 & 73 & Female & Unruptured & Right & 11.0 & - & Yes & Superior thyroid artery & C1, PcomA & 506 & - & ATPA infarction & 0 \\
\hline 3 & 75 & Female & Unruptured & Right & 16.8 & - & Yes & Superior thyroid artery & C1, PcomA & 283 & - & No lesion & 0 \\
\hline 4 & 81 & Female & Unruptured & Left & 10.6 & - & No & Superior thyroid artery & $\mathrm{A} 1, \mathrm{M} 1$ & 605 & Myocardial infarction & No lesion & 1 \\
\hline 5 & 71 & Female & Unruptured & Right & 17.8 & $\begin{array}{l}\text { Anterior clinoidectomy, } \\
\text { STA-MCA bypass, } \\
\text { ECA-RA-M2 bypass }\end{array}$ & Yes & Cervical ICA & A1, M1, PcomA & 820 & - & ATPA infarction & 0 \\
\hline 6 & 71 & Female & Unruptured & Right & 14.9 & - & No & Superior thyroid artery & C1, PcomA & 814 & - & No lesion & 0 \\
\hline 7 & 65 & Female & Unruptured & Left & 10.4 & - & No & Superior thyroid artery & $\mathrm{A} 1, \mathrm{M} 1$ & 363 & - & No lesion & 0 \\
\hline 8 & 54 & Female & Unruptured & Left & 18.0 & - & No & Superior thyroid artery & C1, PcomA & 430 & - & No lesion & 0 \\
\hline 9 & 82 & Female & Unruptured & Right & 14.1 & - & Yes & Superior thyroid artery & A1, M1, PcomA & 452 & - & No lesion & 0 \\
\hline 10 & 73 & Female & Unruptured & Left & 10.7 & - & Yes & Superior thyroid artery & C1, PcomA & 444 & - & No lesion & 0 \\
\hline 11 & 85 & Female & Unruptured & Left & 12.5 & - & Yes & Superior thyroid artery & C1, PcomA & 448 & - & No lesion & 0 \\
\hline 12 & 53 & Female & Unruptured & Right & 18.0 & Anterior clinoidectomy & No & Superior thyroid artery & C1, PcomA & 430 & - & No lesion & 0 \\
\hline 13 & 76 & Female & Unruptured & Right & 24.1 & $\begin{array}{l}\text { Anterior clinoidectomy, } \\
\text { STA-MCA bypass, } \\
\text { ECA-RA-M2 bypass }\end{array}$ & No & Cervical ICA & C1, PcomA & 1126 & Paresis, memory disturbance & AchA infarction & 3 \\
\hline 14 & 71 & Male & Unruptured & Right & 8.6 & - & No & Superior thyroid artery & C1, PcomA & 528 & - & No lesion & 0 \\
\hline 15 & 52 & Female & $\begin{array}{l}\text { Ruptured } \\
\text { (Hunt and } \\
\text { Hess grade 2) }\end{array}$ & Left & 9.6 & STA-MCA bypass & Yes & Superior thyroid artery & C1, PcomA & 864 & - & No lesion & 0 \\
\hline 16 & 70 & Female & Unruptured & Right & 8.7 & - & No & Superior thyroid artery & $\mathrm{C} 1$ & 361 & - & No lesion & 0 \\
\hline 17 & 62 & Female & Unruptured & Left & 38.0 & $\begin{array}{l}\text { Anterior clinoidectomy, } \\
\text { STA-MCA bypass, } \\
\text { ECA-RA-M2 bypass }\end{array}$ & No & Cervical ICA & $\mathrm{C} 1$ & 894 & Transient memory disturbance & ATPA infarction & 1 \\
\hline 18 & 69 & Male & Unruptured & Left & 12.7 & $\begin{array}{l}\text { Anterior clinoidectomy, } \\
\text { STA-MCA bypass }\end{array}$ & Yes & Superior thyroid artery & C1, PcomA & 960 & - & No lesion & 0 \\
\hline 19 & 66 & Male & Unruptured & Left & 11.5 & - & Yes & Superior thyroid artery & C1, PcomA & 520 & Transient memory disturbance & ATPA infarction & 1 \\
\hline 20 & 75 & Female & Unruptured & Right & 11.1 & - & No & Superior thyroid artery & $\mathrm{C} 1$ & 373 & Transient paresis & ATPA infarction & 1 \\
\hline
\end{tabular}



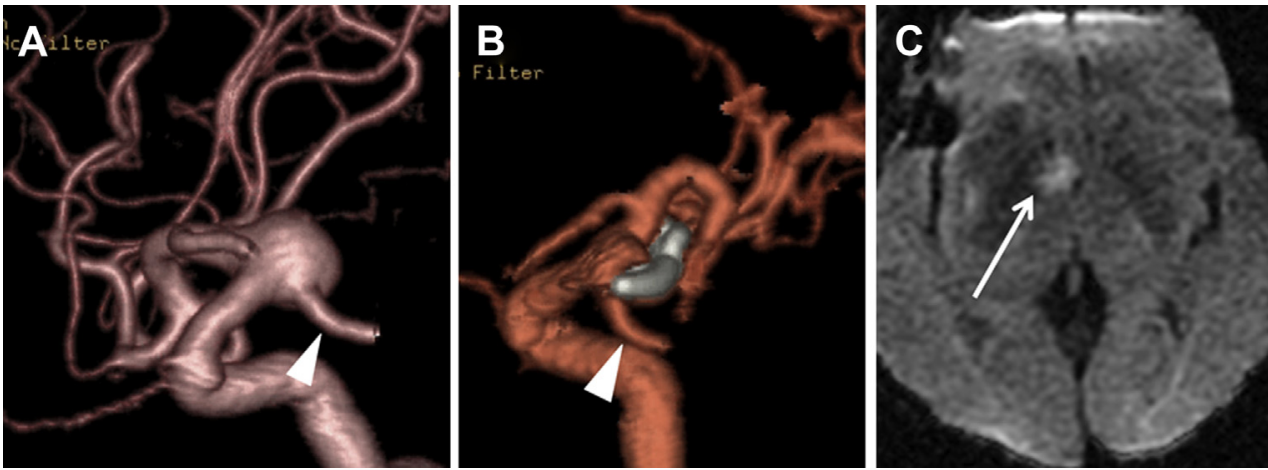

Figure 5. Representative preoperative three-dimensional computed tomography (3D-CT) angiograms and postoperative magnetic resonance images in a patient who developed postoperative cerebral ischemia (patient 2). (A) Preoperative 3D-CT angiogram (anteromedial view) showing a right internal carotid-posterior communicating artery (arrowhead) aneurysm (11 mm in size). (B) Postoperative 3D-CT angiogram (posteromedial view) showing complete occlusion of the aneurysm with preservation of the PcomA (arrowhead). (C) Postoperative diffusion-weighted magnetic resonance image showing a small right infarction (arrow) in the territory of the right anterior thalamoperforating artery.

high blood pressure, and familial history of intracranial aneurysms, and the findings of the Unruptured Cerebral Aneurysm Study in Japan demonstrating that ICA-PcomA aneurysms are more likely to rupture, leading to recommendation for treatment (clipping or coiling). ${ }^{\mathrm{I}}$

Careful study of preoperative images is important for the planning of surgery for $\mathrm{CI}$ aneurysms. The relationship between the aneurysm and the ipsilateral PcomA should be carefully considered, as 15 of 20 cases in our series required temporary occlusion of the PcomA to reduce retrograde blood flow into the aneurysm sac. In addition, the occlusion period should be limited to Io minutes per attempt to decrease the risk of cerebral ischemia. RSD can provide a wide operative field to prevent inadvertent injury of small perforators, which tend to be located behind the aneurysm in most cases. We have routinely used the anterior temporal approach to provide a wide operative field in the retrocarotid space in cases of $\mathrm{CI}$ aneurysms. ${ }^{\mathrm{I5}, \mathrm{I7}, \mathrm{I8}}$ We recommend taking precautions, including complete hemostasis before further steps, to avoid blood obscuring the surgical field. Furthermore, the surgeon and the assistant must closely synchronize their actions to shorten the occlusion time when using the RSD technique.

\section{Clinical Outcome and Complications}

Good outcomes for RSD-assisted clipping of CI aneurysms were previously reported in $79 \%$ to $83 \%$ of patients ${ }^{6,7}$ similar to our results that demonstrated good outcomes in $95 \%$ of cases. RSDassisted clipping of large or giant $\mathrm{C}_{2} / \mathrm{C}_{3}$ aneurysms has been associated with visual neurologic deficits. ${ }^{\mathrm{IO}}$ In contrast, our study showed no visual worsening after RSD-assisted clipping.

In our series, postoperative ischemic lesions were mostly asymptomatic or transient, and relatively common in the territories of the AchA and ATPA, and mostly related to the size (large or giant) of the $\mathrm{CI}_{\mathrm{I}}$ aneurysm; however, this is unsurprising because the reported postoperative morbidity rate for large or giant $\mathrm{CI}_{\mathrm{I}}$ aneurysms is $36 \%-67 \%$ of cases. ${ }^{19,20}$ Theoretically, in cases of strong attachments and adherences to the perforators, careful dissection of the aneurysm dome after gentle suction of the aneurysm through the RSD may be helpful to decrease the rate of perforator injury and consequently reduce the rate of ischemic events. However, our study did not assess this aspect. Further large, case-controlled or randomized controlled studies may be required to determine whether the RSD technique can prevent postoperative ischemia in the territory of small perforators.

\section{Bypass Procedures for Large or Giant Aneurysms}

Revascularization procedures or bypass techniques are useful to prevent cerebral ischemia caused by prolonged temporary occlusion during clipping of large or giant aneurysms. ${ }^{21,22}$ This is especially the case with complex aneurysms, in which clipping may require prolonged temporary or permanent occlusion of the parent vessel. ${ }^{23}$ In our series, none of the patients developed bypass-related complications.

\section{Limitations}

This study has certain limitations. First, this was a retrospective case series of $\mathrm{CI}$ aneurysms treated using RSD-assisted clipping. Second, the decision making for the necessity of bypass was based mainly on aneurysm complexity and institutional experience.

\section{CONCLUSIONS}

The RSD technique is a useful procedure to achieve proximal vascular control, to soften and shrinkage the aneurysm sac, and to provide a wide and clean operative field allowing safe clip placement. The RSD technique requires special attention to the relationship between the perforators and the aneurysm, and close cooperation between the surgeon and the assistant. 


\section{REFERENCES}

I. Albert FK, Forsting M, Aschoff A, Krieger D, Kunze S. Clipping of proximal paraclinoid aneurysms with support of the balloon-catheter "trapping-evacuation" technique. Technical note. Acta Neurochir (Wien). I993;I25:I38-I4I

2. Bouthillier A, van Loveren HR, Keller JT. Segments of the internal carotid artery: a new classification. Neurosurgery. I996;38:425-432.

3. Flamm ES. Suction decompression of aneurysms. Technical note. J Neurosurg. I981;54:275-276.

4. Batjer HH, Samson DS. Retrograde suction decompression of giant paraclinoidal aneurysms. Technical note. J Neurosurg. I990;73:305-306.

5. Tamaki N, Kim S, Ehara K, Asada M, Fujita K, Taomoto K, et al. Giant carotid-ophthalmic artery aneurysms: direct clipping utilizing the "trappingevacuation" technique. J Neurosurg. I991;74: 567-572.

6. Chen SF, Kato Y, Subramanian B, Kumar A, Watabe T, Imizu S, et al. Retrograde suction decompression assisted clipping of large and giant cerebral aneurysms: our experience. Minim Invasive Neurosurg. 20II;54:I-4.

7. Eliava SS, Filatov YM, Yakovlev SB, Shekhtman OD, Kheireddin AS, Sazonov IA, et al. Results of microsurgical treatment of large and giant ICA aneurysms using the retrograde suction decompression (RSD) technique: series of $92 \mathrm{pa}$ tients. World Neurosurg. 2010;73:683-687.

8. Fahlbusch R, Nimsky C, Huk W. Open surgery of giant paraclinoid aneurysms improved by intraoperative angiography and endovascular retrograde suction decompression. Acta Neurochir (Wien). I997;I39:1026-I032.

9. Fulkerson DH, Horner TG, Payner TD, Leipzig TJ, Scott JA, Denardo AJ, et al. Endovascular retrograde suction decompression as an adjunct to surgical treatment of ophthalmic aneurysms: analysis of risks and clinical outcomes. Neurosurgery. 2009;64(3 Suppl):ONSio7-ONSIII. ro. Mattingly T, Kole MK, Nicolle D, Boulton M Pelz D, Lownie SP. Visual outcomes for surgical treatment of large and giant carotid ophthalmic segment aneurysms: a case series using retrograde suction decompression (the "Dallas technique") J Neurosurg. 2013;II8:937-946.

II. Mizoi K, Takahashi A, Yoshimoto T, Fujiwara S, Koshu K. Combined endovascular and neurosurgical approach for paraclinoid internal carotid artery aneurysms. Neurosurgery. I993;33:086-992.

I2. Scott JA, Horner TG, Leipzig TJ. Retrograde suction decompression of an ophthalmic artery aneurysm using balloon occlusion. Technica note. J Neurosurg. I991;75:I46-I47.

13. Szmuda T, Sloniewski P. Early and long-term outcome of surgically treated giant internal carotid artery aneurysms-comparison with smalle aneurysms. Acta Neurochir (Wien). 20II;153: I6II-I6I9.

I4. Matsukawa $\mathrm{H}$, Tanikawa $\mathrm{R}$, Kamiyama $\mathrm{H}$ Tsuboi T, Noda K, Ota N, et al. Localization in the interpeduncular cistern as risk factors for the thalamoperforators' ischemia, poor outcome, and oculomotor nerve palsy in patients with complex unruptured basilar apex aneurysm treated with neck clipping. World Neurosurg. 2015;84:475-482.

I5. Takeuchi S, Tanikawa R, Tsuboi T, Noda K, Oda J, Miyata S, et al. Superficial temporal artery to proximal posterior cerebral artery bypass through the anterior temporal approach. Surg Neurol Int. 20I5;6:95.

I6. Morita A, Kirino T, Hashi K, Aoki N, Fukuhara S, Hashimoto $\mathrm{N}$, et al. The natural course of unruptured cerebral aneurysms in a Japanese cohort. N Engl J Med. 20I2;366:2474-2482.

I7. Heros RC, Lee SH. The combined pterional/ anterior temporal approach for aneurysms of the upper basilar complex: technical report. Neurosurgery. I993;33:244-250.

I8. Katsuno M, Tanikawa R, Miyazaki T, Ota N, Noda K, Izumi N, et al. The limits and countermeasures of the anterior temporal approach for unruptured upper basilar artery aneurysms [in Japanese] No Shinkei Geka. 20I3;4I:3II-3I8.

I9. Takebayashi S, Kamiyama H, Takizawa K Kobayashi T, Saitoh N. The significance of intraoperative monitoring of muscle motor evoked potentials during unruptured large and giant cerebral aneurysm surgery. Neurol Med Chir (Tokyo). 20I4;54:I80-I88.

20. Velat GJ, Zabramski JM, Nakaji P, Spetzler RF. Surgical management of giant posterior communicating artery aneurysms. Neurosurgery. 2012;7I(I Suppl Operative):43-50.

2I. Sanai N, Zador Z, Lawton MT. Bypass surgery for complex brain aneurysms: an assessment of intracranial-intracranial bypass. Neurosurgery. 2009;65:670-683.

22. Sanai N, Lawton MT. Microsurgical managemen of giant intracranial aneurysms. In: Winn HR, ed. Youmans Neurological Surgery. 6th ed. 4. Philadelphia: Elsevier-Saunders; 2011:3953-3971.

23. Ishishita $\mathrm{Y}$, Tanikawa $\mathrm{R}$, Noda $\mathrm{K}$, Kubota $\mathrm{H}$ Izumi N, Katsuno M, et al. Universal extracranialintracranial graft bypass for large or giant interna carotid aneurysms: techniques and results in 38 consecutive patients. World Neurosurg. 20I4;82: I30-I39.

Conflict of interest statement: The authors report no conflict of interest concerning the materials or methods used in this study or the findings specified in this paper.

Received 28 September 2015; accepted 26 December 2015

Citation: World Neurosurg. (2016) 89:19-25.

http://dx.doi.org/10.1016/j. wneu.2015.12.095

Journal homepage: www.WORLDNEUROSURGERY.org

Available online: www.sciencedirect.com

1878-8750/\$ - see front matter (C) 2016 Elsevier Inc. All rights reserved. 